\section{Research Square}

Preprints are preliminary reports that have not undergone peer review.

They should not be considered conclusive, used to inform clinical practice, or referenced by the media as validated information.

\title{
Safety of PRRSV-2 Vaccines Vaccination via Intramuscularly or Intradermally Routes and Evaluation of PRRSV Transmission and the Induction of Immune Response Between Needle- Free and Conventional Needle
}

Adthakorn Madapong

Chulalongkorn University

Kepalee Saeng-chuto

Chulalongkorn University

Angkana Tantituvanont

Chulalongkorn University

Dachrit Nilubol ( $\sim$ dachrit@gmail.com)

Chulalongkorn University

\section{Research Article}

Keywords: PRRSV-2 vaccines, intramuscularly or intradermally routes, evaluation of PRRSV, immune response, needle-free, conventional needle, Safety

Posted Date: June 2nd, 2021

DOI: https://doi.org/10.21203/rs.3.rs-568199/v1

License: (9) (1) This work is licensed under a Creative Commons Attribution 4.0 International License. Read Full License 


\section{Abstract}

Two separated experiments (Exp) were conducted to evaluate the shedding and efficacy of 2 modified live porcine reproductive and respiratory syndrome virus (PRRSV) type 2 vaccines (MLV) when administered intramuscularly (IM) or intradermally (ID) (Exp A), and the potential of PRRSV transmission using a needle-free device (Exp B). 154, castrated-male, pigs were procured from a PRRSV-free herd. In Exp A, 112 pigs were randomly allocated into 4 groups of 21 pigs including IM/Ingelvac MLV (G1), IM/Prime Pac (G2), ID/Prime Pac (G3), and non-vaccination (G4). G1 was IM vaccinated once with Ingelvac PRRS MLV (Ing) (Boehringer Ingelheim, Germany). G2 and G3 were IM and ID vaccinated once with Prime Pac PRRS (PP) (MSD Animal Health, The Netherlands), respectively. Following vaccination, an antibody response, IFN- $\gamma-S C$, and IL-10 were monitored. Sera, tonsils, nasal swabs, bronchoalveolar lavage (BAL), urines, and feces were collected from 3 vaccinated pigs each week to 42 days post-vaccination (DPV) and assayed for the presence of PRRSV using virus isolation and PCR. Age-matched sentinels were introduced weekly into vaccinated groups from 0 to 42 DPV and monitored for seroconversion. In Exp B, pigs were randomly allocated into 5 groups of 3 pigs each including IM/High (T1), ID/High (T2), IM/Low (T3), ID/Low (T4), and NoChal. The T1 and T2, and T3 and T4 groups were intranasally challenged with HP-PRRSV-2 at high $\left(10^{6}\right)$ and low $\left(10^{3} \mathrm{TCID}_{50} / \mathrm{ml}\right)$ doses, respectively. At 7 days post-challenge (DPC), T1 and T2, and T3 and T4 groups were IM and ID injected with Diluvac Forte using needles and a needless device (IDAL 3G, MSD Animal Health, The Netherlands), respectively. Same needles or devices were used to inject the same volume of Diluvac Forte into sentinel pigs. Seroconversion of sentinels was evaluated. The results demonstrated that PP vaccinated groups (G2 and G3), regardless of the route of vaccination, had ELISA response significantly lower than G1 at 7 and 14 DPV. PP-vaccinated groups (G2 and G3) had significantly higher IFN- - -SC and lower IL-10 compared to the Ing-vaccinated group (G1). Based on IM, 2 different MLV had different virus distribution and shedding patterns. PP-vaccinated pigs had significantly shortened viremia than the Ingvaccinated pigs. However, ID-vaccinated pigs had lower virus distribution in organs without virus shedding to sentinel pigs. In Exp B, ID-injected sentinel pigs had no seroconversion compared to IM-injected sentinel pigs regardless of the challenge dose. In conclusion, our results demonstrated that ID vaccination might represent an alternative to improve vaccine efficacy and safety, and may be able to reduce the shedding of vaccine viruses and reduce the iatrogenic transfer of pathogens between animals with shared needles.

\section{Introduction}

Porcine reproductive and respiratory syndrome (PRRS) is an economically devastating disease in pigs characterized by respiratory distress in finishing pigs and reproductive disorders in breeding dams ${ }^{1,2}$. PRRS virus (PRRSV), an enveloped, positive-sense, single-stranded RNA virus belonged to the order Nidovirales, family Arteriviridae is the causative agent ${ }^{3}$. PRRSV has been classified into two genetically distinct species including PRRSV- 1 and PRRSV- ${ }^{4}$. PRRSV-1 has predominantly existed in European countries and currently has further evolved into 4 subtypes ${ }^{5}$. Meanwhile, PRRSV-2 has been dominant in North American continent ${ }^{6}$ and has further evolved into 9 distinct lineages ${ }^{7}$.

Since its first emergence in the late 1980's, PRRSV continues to cause economic losses to swine production worldwide. To control PRRS, several types of vaccines including inactivated, modified live vaccine (MLV) and subunit vaccines have been implemented in swine production worldwide with varying degree of success. Presently, a modified-live vaccine (MLV) has been deemed to be the most efficacious against PRRSV infection and has been employed regularly in both experimental and field-scale trials since its first launch in 1994. Several PRRSV MLVs, both PRRSV-1 and PRRSV-2, have been licensed in various countries depending on circulating PRRSV genotypes. However, concerns regarding the safety of PRRSV MLV has been raised by some studies demonstrating the shedding and persistence of vaccine virus in vaccinated hosts, in turn causing detectable viremia and potential transmission of vaccine virus to naïve animals ${ }^{8,9}$. In addition, the vaccine virus can cross the placental barrier in pregnant sows and infect the developing fetuses ${ }^{10}$ resulting in the transmission to naïve newborn piglets during lactation ${ }^{1}$. Finally, vaccine virus has been demonstrated to have a potential to merge with field virus in a recombination event, generating potential new genetically distinct variants of PRRSV in the farm that may contribute to virulence and disease incidence ${ }^{11}$. To deal with these potential issues, it is necessary to choose a PRRSV MLV that potentially has the least level of shedding and persistence of vaccine virus.

Intramuscular administration using needles has been the main route of vaccination in pigs. However, risks associated with needles have been increased. Therefore, alternative routes of vaccine administration are urgently considered. Needle-free devices have been used in human medicine to deliver antigen into skin for sometimes ${ }^{12}$. Antigen delivery using needle free devices can be performed intradermally or transdermally. Presently, vaccination using needle-free devices is commercially available in pigs and intradermal vaccination of pigs has been shown to be able to trigger adaptive and humoral responses, even in the absence of adjuvant ${ }^{13}$. Currently, several commercially available vaccines against swine-relevant pathogens [i.e., Mycoplasma hyopneumoniae, PRRSV, porcine circovirus 2 , and pseudorabies virus] are licensed for the intradermal needle-free delivery route ${ }^{14}$. Apart from animal welfare benefits, intradermal needle-free vaccination has the potential to eliminate accidental needle-stick injuries by farm workers, reduce iatrogenic disease transfer from shared needles and reduce injection site lesions caused by intramuscular vaccination, which are known to be prevalent in swine at the slaughterhouse ${ }^{15}$. Additionally, it is also known that PRRSV can be transmitted through iatrogenic transfer between pigs when needles are reused during routine vaccination of PRRSV ${ }^{16}$.

Different PRRSV-2 MLV vaccines have been increasingly used in swine herds in South East Asian countries. There are variety of vaccine delivery routes and commercial vaccines, and there is also increased use of intradermal needle-free delivery devices to administer PRRSV-2 MLV to pigs. However, there are only few reports that compare the safety of different PRRSV-2 MLVs, as well as reports that study the ability of needle-free devices to reduce iatrogenic disease transfer via needles. Gathering together, a safe PRRSV-2 MLV delivered through a needle-free jet injector could potentially thus reduce virus shedding and persistence in the environment, as well as iatrogenic transfer of wild type PRRSV between vaccinated pigs.

Therefore, the objectives of the present study were to investigate safety issues, in terms of viral persistence in tissues and vaccine virus shedding to sentinel pigs, of 2 commercially available PRRSV-2 MLVs administered intramuscularly and intradermally. The antibody response, interferon- $\gamma$-secreting cells (IFN- $\gamma$ SC), IL-10 production were additionally observed. Moreover, the potential transmission of PRRSV between the needle-free device and the conventional needle was investigated. 


\section{Materials And Methods}

Ethical Statement for experimental procedures. All animal procedures were conducted following the recommendations in the Guild for the Care and Use of Laboratory Animal of the National Research Council of Thailand according to protocols reviewed and approved by the Chulalongkorn University Animal Care and Use Committee under protocol number 2031015, animal use license number UI-00058-2558. All methods were performed in accordance with the relevant guidelines and regulations. The study is reported in accordance with the ARRIVE guidelines (https://arriveguidelines.org).

PRRSV vaccines, vaccination, and viruses. PRRSV vaccines used for vaccination were 2 PRRSV-2 MLVs including Ingelvac PRRS MLV (Boehringer Ingelheim, Rhein, Germany) and Prime Pac PRRS (MSD Animal Health, Boxmeer, The Netherlands), respectively. Prime Pac PRRS is available in 2 different preparations, intramuscular (IM) and intradermal (ID) vaccination. Dosage and administration routes were following the manufacturer's instructions. In brief, a 2 ml and 1 $\mathrm{ml}$ dose of Ingelvac PRRS MLV (batch no2451218A) and Prime Pac PRRS (batch no. A065CE04) was used for IM vaccination, respectively. A 0.2 ml dose of Prime Pac PRRS (batch no. A065CE04) was used for ID vaccination. ID vaccination and injection were performed using IDAL 3G needle-free device (MSD Animal Health, Boxmeer, The Netherlands).

Homologous vaccine viruses and highly pathogenic (HP)-PRRSV-2 were used in the present study. Homologous vaccine viruses refer to vaccine strains that were used as recall antigens for in vitro stimulation assay in the measurement of IFN- $\gamma-S C$ and IL-10 production, as previously described methods ${ }^{17}$. To challenge pigs, the FDT10uS23 isolate was used as a virus inoculum at the fifth passage in MARC-145 cells. Thai PRRSV-2 (HP-PRRSV-2,) was used. The FDT10US23 isolate is a HP-PRRSV-2 variant genetically classified in sublineage 8.7/HP-PRRSV-2 based on international systematic classification according to the previously described method ${ }^{7}$. The ORF5 genome sequence is available in GenBank under accession number JN255836. This isolate was isolated from swine herds experiencing PRRS outbreaks in the western region of Thailand during 2010-2011 ${ }^{18}$. Pathogenesis and challenge studies of the challenged isolate were demonstrated according to previous studies ${ }^{18-22}$.

Experimental design. One hundred fifty-four, castrated-male, PRRSV-free pigs at three weeks of age were procured from a PRRSV-free herd. Upon arrival, sera were collected individually and assayed for the presence of viral RNA and PRRSV-specific antibodies using PCR and ELISA to confirm their negative status. In the present study, two separate experiments were conducted. In experiment A (Exp A), pigs were vaccinated once with PRRSV-2 MLV via intramuscular (IM) or intradermal (ID) routes. PRRSV-specific antibodies and cell-mediated were evaluated. The presence of the vaccine virus in tissues and organs and the shedding pattern to sentinel pigs were determined. In experiment B (Exp B), pigs were intranasally challenged with HP-PRRSV-2. At 7 days post-challenge (DPC), the highest level of viremia, challenged pigs were injected with Diluvac Forte, either IM or ID route, using a conventional needle or needle-free device, respectively. The same needle or needle-free device was used to inject the Diluvac Forte into sentinel pigs. Seroconversion of the sentinel pigs was determined. Pigs in each group were kept in separate rooms with separated air spaces and monitored daily for physical condition and clinical respiratory disease throughout the experiment.

Experiment A. One hundred and twelve pigs were randomly allocated based on weight stratification into 4 groups of 21 pigs each including IM/Ingelvac MLV (G1), IM/Prime Pac (G2), ID/Prime Pac (G3), and NoVac (G4) as showed in Table 1. The IM/Ingelvac MLV (G1) group was IM vaccinated once with a 2 ml dose of Ingelvac PRRS MLV (Boehringer Ingelheim, Rhein, Germany). The IM/Prime Pac (G2) and ID/Prime Pac (G3) groups were vaccinated once via IM and ID routes with a $1 \mathrm{ml}$ and $0.2 \mathrm{ml}$ dose of Prime Pac PRRS (MSD Animal Health, Boxmeer, The Netherlands), respectively. ID vaccination was performed using IDAL $3 \mathrm{G}$ needle-free vaccinator. The NoVac (G4) group was left non-vaccination and the remaining 38 pigs were served as age-matched sentinel pigs.

Table 1

Experimental design of experiment A (Exp A). Four treatment groups included 3 vaccinated groups and 1 non-vaccinated group, respectively. Routes of vaccine administration are included either intramuscular (IM) or intradermal (ID). ID vaccination was performed using IDAL $3 \mathrm{G}$ needle-free device.

\begin{tabular}{|lllllll|}
\hline Treatment groups & $\begin{array}{l}\text { Number of } \\
\text { pigs }\end{array}$ & Vaccination & Vaccines & $\begin{array}{l}\text { Vaccine } \\
\text { type }\end{array}$ & $\begin{array}{l}\text { Dosage and route of } \\
\text { administration }\end{array}$ & Manufacturers \\
\hline $\begin{array}{l}\text { IM/Inglevac MLV } \\
\text { (G1) }\end{array}$ & 21 & Yes & $\begin{array}{l}\text { Ingelvac PRRS } \\
\text { MLV }\end{array}$ & PRRSV-2 & 2 ml, IM & $\begin{array}{l}\text { Boehringer Ingelheim, Rhein, } \\
\text { Germany }\end{array}$ \\
$\begin{array}{l}\text { IM/Prime Pac } \\
\text { (G2) }\end{array}$ & 21 & Yes & $\begin{array}{l}\text { Prime Pac } \\
\text { PRRS }\end{array}$ & PRRSV-2 & $1 \mathrm{ml}$, IM & $\begin{array}{l}\text { MSD Animal Health, Boxmeer, The } \\
\text { Netherlands }\end{array}$ \\
\hline $\begin{array}{l}\text { ID/Prime Pac } \\
(\text { G3) }\end{array}$ & 21 & Yes & $\begin{array}{l}\text { Prime Pac } \\
\text { PRRS }\end{array}$ & PRRSV-2 & $0.2 \mathrm{ml}$ ID & $\begin{array}{l}\text { MSD Animal Health, Boxmeer, The } \\
\text { Netherlands }\end{array}$ \\
\hline NoVac (G4) & 21 & No & - & - & - & - \\
\hline
\end{tabular}

Blood samples were collected at $0,7,14,21,28,35,42,49,56$, and 63 days post-vaccination (DPV). Sera were assayed for PRRSV specific antibody and PRRSV RNA using ELISA and quantitative RT-qPCR (qPCR), respectively. Peripheral blood mononuclear cells (PBMC) were isolated and used for in vitro stimulation to measure IL-10 production using an ELISA kit and IFN- $\gamma-S C$ using an ELISPOT assay. Three pigs from each group were necropsied on weekly basis starting from 7 to 42 DPV. Sera, nasal swabs, tonsils, lungs, bronchoalveolar lavages (BAL), and feces were collected and assayed for the presence of PRRSV using qPCR and virus isolation in cell culture. The shedding of vaccine virus to naïve animals was measured by placing age-matched sentinel pigs in contact with the vaccinated pigs at 1, 7, 14, 21, 28, 35, and 42 DPV. Sentinel pigs were comingled with vaccinated pigs for 3 consecutive weeks. Sera were collected at 0 , and 21 days post commingle. Commingled pigs were weekly measured PRRSV specific antibody and PRRSV RNA using ELISA and qPCR, respectively. 
Experiment B. Forty-two pigs were randomly allocated based on weight stratification into 5 groups with 3 pigs each including IM/High (T1), ID/High (T2), IM/Low (T3), ID/Low (T4), and NoChal (T5) as showed in Table 2. Two different dosages of HP-PRRSV-2 were used to inoculate pigs. The IM/High (T1) and ID/High (T2) groups were intranasally inoculated with $4 \mathrm{ml}$ (2 ml/nostril) of HP-PRRSV-2 (FDT10US23 isolate, $10^{6}$ TCID $50 / \mathrm{ml}$ ). The IM/Low (T3) and ID/Low (T4) groups were intranasally inoculated with $4 \mathrm{ml}$ of HP-PRRSV-2 at a lower dose (FDT10uS23 isolate, $10^{3} \mathrm{TCID}_{50} / \mathrm{ml}$ ). The NoChal (T5) group was served as a control, and the remaining 17 pigs were used as age-matched sentinel pigs. Sera were collected at $0,7,14,21$, and 28 DPC and assayed for the presence of PRRSV-specific antibody and RNA using ELISA and qPCR, respectively.

Table 2

Experimental design of experiment B (Exp B). Five treatment groups included 4 challenged groups and 1 non-challenged group, respectively. Pigs were intranasally inoculated with HP-PRRSV-2 at 0 days post-challenge (DPC). Pigs in non-challenge (NoChal) served as the control. At 7 DPC, the challenged pigs were injected with Diluvac Forte via either IM or ID routes. ID injection was performed using IDAL $3 G$ needle-free device.

\begin{tabular}{|c|c|c|c|c|c|c|}
\hline $\begin{array}{l}\text { Treatment } \\
\text { groups }\end{array}$ & $\begin{array}{l}\text { Number of } \\
\text { pigs }\end{array}$ & Challenge & Detail & $\begin{array}{l}\text { Dosage and challenge } \\
\text { route }\end{array}$ & PRRSV isolate & $\begin{array}{l}\text { Diluvac Forte } \\
\text { injection }\end{array}$ \\
\hline IM/High (T1) & 3 & Yes & $\begin{array}{l}\text { High dose group for IM } \\
\text { injection }\end{array}$ & $\begin{array}{l}4 \mathrm{ml} / \mathrm{pig} \text {, at } 10^{6} \\
\mathrm{TCID}_{50} / \mathrm{ml}\end{array}$ & $\begin{array}{l}\text { HP-PRRSV-2 } \\
\text { (FDT10US23) }\end{array}$ & $1 \mathrm{ml} /$ pig, IM \\
\hline ID/High (T2) & 3 & Yes & $\begin{array}{l}\text { High dose group for ID } \\
\text { injection }\end{array}$ & $\begin{array}{l}4 \mathrm{ml} / \mathrm{pig} \text {, at } 10^{6} \\
\operatorname{TCID}_{50} / \mathrm{ml}\end{array}$ & $\begin{array}{l}\text { HP-PRRSV-2 } \\
\text { (FDT10US23) }\end{array}$ & $0.2 \mathrm{ml} / \mathrm{pig}, \mathrm{ID}$ \\
\hline IM/Low (T3) & 3 & Yes & $\begin{array}{l}\text { Low dose group for IM } \\
\text { injection }\end{array}$ & $\begin{array}{l}4 \mathrm{ml} / \mathrm{pig}, \text { at } 10^{3} \\
\mathrm{TCID}_{50} / \mathrm{ml}\end{array}$ & $\begin{array}{l}\text { HP-PRRSV-2 } \\
\text { (FDT10US23) }\end{array}$ & $1 \mathrm{ml} /$ pig, IM \\
\hline ID/Low (T4) & 3 & Yes & $\begin{array}{l}\text { Low dose group for ID } \\
\text { injection }\end{array}$ & $\begin{array}{l}4 \mathrm{ml} / \mathrm{pig} \text {, at } 10^{3} \\
\mathrm{TCID}_{50} / \mathrm{ml}\end{array}$ & $\begin{array}{l}\text { HP-PRRSV-2 } \\
\text { (FDT10US23) }\end{array}$ & $0.2 \mathrm{ml} / \mathrm{pig}, \mathrm{ID}$ \\
\hline NoChal (T5) & 3 & No & Negative control & - & - & - \\
\hline
\end{tabular}

At 7 DPC, the IM/High (T1) and IM/Low (T3) groups were IM injected with $1 \mathrm{ml}$ of Diluvac Forte (batch no G197A01, MDS Animal Health, Boxmeer, The Netherlands) using a conventional needle (G18, 1"). The ID/High (T2) and ID/Low (T4) groups were ID injected with $0.2 \mathrm{ml}$ of Diluvac Forte using IDAL $3 G$ needle-free vaccinator (MSD Animal Health, Boxmeer, The Netherlands). The same conventional needles or needle-free device were used to inject the same volume of Diluvac Forte (MSD Animal Health, Boxmeer, The Netherlands) to sentinel pigs with the same route of injection (1 injected pig to 2 sentinels). The NoChal group was left as a negative control. Blood samples were collected from sentinel pigs at $0,7,14,21$, and 28 days post-injection (DPI). Injected sentinel pigs were weekly measured PRRSV-specific antibody and PRRSV RNA using ELISA and qPCR, respectively.

Clinical evaluation. Clinical signs were monitored daily post-vaccination and post-challenge periods for two consecutive weeks by the same person at the same time. The severity of clinical respiratory disease for each pig was evaluated using a scoring system following stress induction as previously described criteria $^{23}: 0=$ normal, 1 = mild dyspnea and/or tachypnea when stressed, $2=$ mild dyspnea and/or tachypnea when at rest, $3=$ moderate dyspnea and/or tachypnea when stressed, 4 = moderate dyspnea and/or tachypnea when at rest, 5 = severe dyspnea and/or tachypnea when stressed, and 6 = severe dyspnea and/or tachypnea when at rest.

Clinical sample collection. Blood was collected from pigs in serum separation tubes (Monovette, Sarstedt, Numbrecht, Germany) and centrifuged at 2,000.g for $10 \mathrm{~min}$ at $20^{\circ} \mathrm{C}$. Sera were stored in $1 \mathrm{ml}$ aliquots at $-80^{\circ} \mathrm{C}$ until used. Nasal swabs, tonsils, and feces were collected using individually packaged sterile swabs which were placed in Dulbecco's modified eagle's medium (DMEM, Gibco, MA, USA) supplemented with 5. antibiotics (100• Antibiotic-antimycotics, Gibco, MA, USA). Tonsils and feces were weighed and mixed with DMEM medium (10\% weight by volume). Then, samples were homogenized and centrifuged at $4,000 \cdot \mathrm{g}$ for $10 \mathrm{~min}$. Homogenates were filtered through $0.2 \mu \mathrm{m}$ pore size filters, treated with 5 . antibiotics overnight at $4{ }^{\circ} \mathrm{C}$, and kept at $-80{ }^{\circ} \mathrm{C}$ until used. Urines were collected from bladders ( $10 \mathrm{ml} / \mathrm{pig}$ ), filtered through $0.2 \mu \mathrm{m}$ pore size filters, and kept at $-80^{\circ} \mathrm{C}$ until used. Bronchoalveolar lavage (BAL) was performed aseptically at necropsy as previously described ${ }^{24}$. In brief, $50 \mathrm{ml}$ of lavage fluid consisting of DMEM with 5 . antibiotics was gently dispensed and aspirated several times into the lungs. The BAL was kept at $-80^{\circ} \mathrm{C}$ until used.

Antibody detection. PRRSV-specific antibodies were measured using a commercial ELISA kit (IDEXX PRRS X3 Ab test, IDEXX Laboratories Inc., MA, USA). The assay was performed following the manufacturer's recommendation. Sera were considered positive for PRRSV antibody if the S/P ratio was greater than 0.4 .

Peripheral blood mononuclear cells (PBMC) isolation. Peripheral blood mononuclear cells (PBMC) were isolated from heparinized blood using gradient density centrifugation (Lymphosep, Biowest, MO, USA) as previously described ${ }^{25}$. Isolated PMBC were counted by an inverted microscope, and concentrations were accessed in cRPMI-1640 [RPMI-1640 media supplemented with 10\% fetal bovine serum (FBS), $2 \mathrm{mM} \mathrm{L-glutamine,} \mathrm{and} 50 \mu \mathrm{g} / \mathrm{ml}$ of gentamycin]. The viability of isolated PBMC was determined by Trypan blue (Sigma-Aldrich, MO, USA) staining and more than $90 \%$ viability were used for in vitro stimulation for IL-10 production and enzyme-linked immunospot (ELISPOT) assay as described below.

Quantification of porcine IL-10. Following vaccination, porcine IL-10 concentration in the supernatant of stimulated PBMC was quantified using a porcine ELISA IL-10 kit (R\&D Systems, MN, USA) under the manufacturer's instructions. In brief, 2 × $10^{6}$ PBMC were seeded into 96-well plates and cultured in vitro for $24 \mathrm{~h}$ with either homologous viruses at 0.01 multiplicity of infection (MOI) or phytohemagglutinin (PHA, $10 \mu \mathrm{g} / \mathrm{ml}$, Sigma-Aldrich, MO, USA). 
ELISPOT assay. The number of PRRSV-specific IFN-y-SC were determined in PBMC using commercial ELISPOT IFN-y kit (ELISpot porcine IFN-y, R\&D Systems, MN, USA), processed according to manufacturer's instruction and previously described ${ }^{19}$. Briefly, $2 \times 10^{5}$ PBMC were seeded into 96 -well plates and stimulated with homologous viruses at $0.01 \mathrm{MOI}$ for $24 \mathrm{~h}$. The linear response was tested between 0.01 to $0.1 \mathrm{MOI}$. Phytohemagglutinin (PHA, $10 \mu \mathrm{g} / \mathrm{ml}$, Sigma-Aldrich, MO, USA) and cRPMI-1640 were used as positive and negative controls, respectively. The spots were counted, and the background values were subtracted from the respective count of the stimulated cells and the immune response was expressed as the number of IFN- $y-S C$ per $10^{6}$ PBMC.

Pathological examination. Three pigs from each vaccinated group were necropsied at 7, 14, 21, 28, 35, and 42 DPV. lung lesions, either macroscopic or microscopic lung lesions associated with PRRSV-induced pneumonia were evaluated as previously described ${ }^{23}$. For macroscopic lung lesions, each lung lobe was assigned a number to reflex the approximate percentage of the volume of the entire lung and the percentage of the volume from each lobe added to the entire lung score (range from 0 to $100 \%$ of the affected lung). Sections were collected from all lung lobes as previously described ${ }^{23}$. Lung tissues were fixed in $10 \%$ neutral buffered formalin for 7 days and routinely processed and embedded in paraffin in an automated tissue processor. Sections were cut at $5 \mu \mathrm{m}$ and stained with hematoxylin and eosin (H\&E). For microscopic lung lesions, the lung sections were examined in a blinded manner and given an estimated score of the severity of interstitial pneumonia. In brief, $0=$ normal; $1=$ mild interstitial pneumonia; $2=$ moderate multifocal interstitial pneumonia; $3=$ moderate diffuse interstitial pneumonia, and $4=$ severe diffuse interstitial pneumonia. The mean values of the microscopic lung lesion score of each group were calculated.

Virus isolation. Virus isolation was performed in MARC-145 and PAM as previously described ${ }^{26}$. In brief, $100 \mu$ l of the filtered clinical sample was incubated in 96-well plates of monolayers of MARC- 145 and PAM for $60 \mathrm{~min}$ at $37^{\circ} \mathrm{C}$ to facilitate adsorption, washed twice with DMEM supplemented with $3 \%$ FBS (Gibco, MA, USA). Then, the plates were incubated for 3 days at $37^{\circ} \mathrm{C}$ in a humidified atmosphere containing $5 \% \mathrm{CO}_{2}$. Media were removed and cells were fixed with a cold acetone-methanol solution for $10 \mathrm{~min}$ and then air-dried. The virus was detected in a monolayer by indirect microscopy using PRRSV-specific monoclonal antibody (mAb) SR-30 (RTI, South Dakota, USA).

Quantification of PRRSV RNA and RT-PCR. Total RNA was extracted from clinical samples using NucleoSpin Virus (Macherey-Nagel, Duren, Germany) according to the manufacturer's instruction. The RNA quality was measured using a NanoDrop spectrophotometer (Colibri spectrometer, Titertek Berthold, Pforzheim, Germany). Copy number of PRRSV RNA in serum was quantified using probed-based real-time PCR as previously described ${ }^{20}$. The reaction was carried out in QuantStudio 3 Real-time PCR machine (Thermo-Fisher Scientific, MA, USA). To detects the presence of virus in clinical samples, extracted RNA was converted into CDNA and used for PCR which was performed using GoTaq Green Master Mix (Promega, WI, USA). Primer specific for the ORF5 gene and detection conditions were followed as previously described ${ }^{17}$.

Statistical analysis. Analysis of variance (ANOVA) was performed to determine if there were significant differences among groups for each day separately. If the $P$-value for an ANOVA table was less than or equal to 0.05 , the difference between treatment groups was evaluated using a multiple comparison test. All data were performed using IBM SPSS Statistic software version 22 (IBM Corporation, WA, USA)

\section{Results}

\section{Experiment A}

Clinical signs. All vaccinated pigs displayed no clinical abnormalities following vaccination, regardless of the route of vaccination.

PRRSV-specific antibody response. Antibody responses between vaccinated groups as measured by ELISA were significantly different between 14 to 21 DPV (Fig. 1A). Antibody response in the IM/Ingelvac MLV (G1) group was first detected at 7 DPV but the level was lower than the cut-off level (S/P ratio < 0.4 ). Then, antibody titers were continually increased and reached above the cut-off level at 14 DPV and remained constant until the end of the experiment. At 14 DPV, the PRRSV specific antibody of the IM/Ingelvac MLV (G1) group had significantly higher titers than the other groups. Whereas the antibody titers of the IM/Prime Pac (G2) and ID/Prime Pac (G3) groups were not different. At 21 DPV, increased antibody titers were detected in the IM/Prime Pac (G2) and ID/Prime Pac (G3) groups, but the ID/Prime Pac (G3) group had antibody titers below the cut-off level. The PRRSV specific antibody titer of the ID/Prime Pac (G3) group was continually increased at $21 \mathrm{DPV}$ and reached above the cut-off level at $28 \mathrm{DPV}$, respectively. There was no difference in the antibody titers among vaccinated groups from 35 to 63 DPV, respectively (Fig. 1A).

Porcine IL-10. Increased IL-10 levels were first detected in all vaccinated groups at 7 DPV, regardless of vaccination routes (Fig. 2). The IM/Ingelvac MLV (G1) group had the significantly highest IL-10 level $(P<0.05)$ than that of other vaccinated groups (range from $8.0 \pm 1.5$ to $32.0 \pm 1.9 \mathrm{pg} / \mathrm{ml}) \mathrm{from} 7$ to $28 \mathrm{DPV}$, respectively. Meanwhile, the ID/Prime Pac (G3) group had the significantly lowest IL-10 level, ranging from $0.8 \pm 1.2$ to $11.0 \pm 1.8 \mathrm{pg} / \mathrm{ml}$, than that of other groups at 7 to 14 DPV, respectively. There was no difference in IL-10 levels between IM/Prime Pac (G2) and ID/Prime Pac (G3) groups from 21 to 42 DPV, respectively.

PRRSV-specific IFN-y-SC. Following vaccination, PRRSV-specific IFN-y-SC was first detected at 21 DPV in all vaccinated groups (Fig. 3). Then, PRRSV-specific IFN-y-SC was gradually increased and reached peaks at 42 DPV in all groups, regardless of the vaccination route. Statistical differences were observed. The ID/Prime Pac (G3) group had significantly $(P<0.05)$ higher IFN- $\gamma-S C$ than that of other groups at 28, 35, and 42 DPV, respectively. At 28 DPV, the IFN-y-SC of the IM/Prime Pac (G2) and IM/Ingelvac MLV (G1) groups showed no difference. Meanwhile, the IM/Ingelvac MLV (G1) groups had significantly $(P<0.05)$ lower IFN-y-SC ( $24 \pm 7$ and $42 \pm 11$ cells/ $10^{6}$ PBMC) than that of the IM/Prime Pac (G2) group (44 \pm 11 and $65 \pm 12$ cells/10 $\left.\mathrm{PBMC}\right)$ at 35 and $42 \mathrm{DPV}$, respectively. 
Viremia. In all vaccinated groups, PRRSV RNA was continually increased and reached peaks at 7 DPV (range from $128.55 \pm 17.28$ to $246.02 \pm 40.30$ copies/ml) and continually decreased to the basal levels from 14 to 21 DPV (range from $29.32 \pm 12.13$ to $51.61 \pm 12.00 \mathrm{copies} / \mathrm{ml}$ ) (Fig. 6A). Then, PRRSV RNA was not detected from 28 to 63 DPV, respectively. PRRSV RNA in all vaccinated groups had significantly higher than in the NoVac (G4) group from 7 to $21 \mathrm{DPV}$. At $7 \mathrm{DPV}$, the IM/Ingelvac MLV (G1) group had significantly higher $(P<0.05)$ PRRSV RNA of $246 \pm 40.30 \mathrm{copies} / \mathrm{ml}$ than in the other vaccinated group. At 14 DPV, the ID/Prime Pac (G3) had significantly lower $(P<0.05)$ PRRSV RNA of $29.32 \pm 12.13$ copies/ml than in the other vaccinated groups. There was no difference in PRRSV RNA among vaccinated groups from 28 to 63 DPV (Fig. 6A).

Macroscopic and microscopic lung lesion. Macroscopic lung lesions were not detected in all vaccinated groups, regardless of the day of necropsy. For microscopic lung lesion score, the lesions were characterized by thickened septa with an increased number of interstitial macrophages and lymphocytes and by type II pneumocyte hyperplasia (Fig. 4). No microscopic lung lesions were detected in ID/Prime Pac and NoVac groups, regardless of the necropsy day. The IM/Ingelvac MLV (G1) group had a significantly $(P<0.05)$ higher microscopic lung lesion score at $0.4 \pm 0.2$ than in the other vaccinated groups at $21 \mathrm{DPV}$ (Fig. 5).

Detection of PRRSV in clinical samples by virus isolation. The results of virus isolation in MARC-145 and PAM cell cultures from different clinical samples collected at different necropsy days from treatment groups are summarized in Table 3. The frequency of PRRSV isolation varied depending on the clinical samples analyzed, in which PRRSV was more frequently isolated from tonsils than from any other samples but was not isolated from the feces and urine in both MARC-145 and PAM cell cultures, regardless of the day of necropsy and vaccine used. Following vaccination, all clinical samples derived from the ID/Prime Pac (G3) and NoVac (G4) groups were negative for PRRSV isolation regardless of the day of necropsy. Whereas PRRSV was detected in clinical samples of the IM vaccinated pigs up to 42 DPV. PRRSV was detected in sera of $3 / 3,3 / 3$, and $2 / 3$ pigs of the IM/Ingelvac MLV (G1) group on 7,14 , and 21 DPV, respectively. Meanwhile, PRRSV was isolated from sera of pigs in the IM/Prime Pac (G2) group at 7 and 14 DPV with $1 / 3$ and $2 / 3$ pigs, respectively. For nasal swab samples, PRRSV was isolated from pigs in the IM/Ingelvac MLV (G1) group at 28, 35 and 42 DPV with 2/3, 2/3, and 1/3 pigs detected, respectively. In contrast, 1 of 3 pigs from the IM/Prime Pac (G2) group had PRRSV-positive sera at 28 DPV only. For tonsils, PRRSV was isolated from 3/3, $3 / 3,2 / 3$, and $2 / 3$ pigs in the IM/Ingelvac MLV (G1) group at 21, 28, and 42 DPV, respectively. In contrast, 1/3, 2/3 and 1/3 pigs in the IM/Prime Pac (G2) group were positive for PRRSV isolation at 21, 28, and 35 DPV, respectively. For the BAL samples, $1 / 3$ and $2 / 3$ pigs in the IM/Ingelvac MLV (G1) group were positive for PRRSV isolation at 21 and 28 DPV, respectively. Whereas PRRSV was detected in the BAL of 1/3 pigs in the IM/Prime Pac (G2) group at 28 DPV only. 
Table 3

Number of PRRSV-positive sample(s) of experiment A (Exp A) using virus isolation in cell culture and PCR. Values expressed as the number of the positive sample(s)/total samples $(n=3)$. BAL: bronchoalveolar lavage; DPV: days post-vaccination; IM: intramuscular; ID: intradermal; PAM: porcine alveolar

\begin{tabular}{|c|c|c|c|c|c|c|c|c|c|c|c|c|c|c|c|c|c|c|c|}
\hline \multirow{2}{*}{$\begin{array}{l}\text { Treatment } \\
\text { groups }\end{array}$} & \multirow[t]{2}{*}{ Samples } & \multicolumn{3}{|c|}{7 DPV } & \multicolumn{3}{|c|}{$14 \mathrm{DPV}$} & \multicolumn{3}{|c|}{$21 \mathrm{DPV}$} & \multicolumn{3}{|c|}{$28 \mathrm{DPV}$} & \multicolumn{3}{|c|}{35 DPV } & \multicolumn{3}{|c|}{42 DPV } \\
\hline & & $V I 1$ & VI2 & $P C R$ & $V I 1$ & VI2 & $P C R$ & VI1 & VI2 & $P C R$ & VI1 & $V I 2$ & $P C R$ & VI1 & VI2 & $P C R$ & VI1 & VI2 & $P C R$ \\
\hline \multirow{6}{*}{$\begin{array}{l}\text { IM/Ingelvac } \\
\text { MLV(G1) }\end{array}$} & Serum & $3 / 3$ & $3 / 3$ & $3 / 3$ & $3 / 3$ & $3 / 3$ & $3 / 3$ & $2 / 3$ & $2 / 3$ & $2 / 3$ & $0 / 3$ & $0 / 3$ & $0 / 3$ & $0 / 3$ & $0 / 3$ & $0 / 3$ & $0 / 3$ & $0 / 3$ & $0 / 3$ \\
\hline & $\begin{array}{l}\text { Nasal } \\
\text { swab }\end{array}$ & $0 / 3$ & $0 / 3$ & $0 / 3$ & $0 / 3$ & $0 / 3$ & $0 / 3$ & $0 / 3$ & $0 / 3$ & $0 / 3$ & $2 / 3$ & $2 / 3$ & $2 / 3$ & $2 / 3$ & $2 / 3$ & $2 / 3$ & $1 / 3$ & $1 / 3$ & $1 / 3$ \\
\hline & Tonsil & $0 / 3$ & $0 / 3$ & $0 / 3$ & $0 / 3$ & $0 / 3$ & $0 / 3$ & $3 / 3$ & $3 / 3$ & $3 / 3$ & $3 / 3$ & $3 / 3$ & $3 / 3$ & $2 / 3$ & $2 / 3$ & $2 / 3$ & $2 / 3$ & $2 / 3$ & $2 / 3$ \\
\hline & BAL & $0 / 3$ & $0 / 3$ & $0 / 3$ & $0 / 3$ & $0 / 3$ & $0 / 3$ & $1 / 3$ & $1 / 3$ & $1 / 3$ & $2 / 3$ & $2 / 3$ & $2 / 3$ & $0 / 3$ & $0 / 3$ & $0 / 3$ & $0 / 3$ & $0 / 3$ & $0 / 3$ \\
\hline & Urine & $0 / 3$ & $0 / 3$ & $0 / 3$ & $0 / 3$ & $0 / 3$ & $0 / 3$ & $0 / 3$ & $0 / 3$ & $0 / 3$ & $0 / 3$ & $0 / 3$ & $0 / 3$ & $0 / 3$ & $0 / 3$ & $1 / 3$ & $0 / 3$ & $0 / 3$ & $0 / 3$ \\
\hline & Feces & $0 / 3$ & $0 / 3$ & $0 / 3$ & $0 / 3$ & $0 / 3$ & $0 / 3$ & $0 / 3$ & $0 / 3$ & $0 / 3$ & $0 / 3$ & $0 / 3$ & $0 / 3$ & $0 / 3$ & $0 / 3$ & $0 / 3$ & $0 / 3$ & $0 / 3$ & $0 / 3$ \\
\hline \multirow{6}{*}{$\begin{array}{l}\text { IM/Prime } \\
\text { Pac (G2) }\end{array}$} & Serum & $1 / 3$ & $1 / 3$ & $1 / 3$ & $2 / 3$ & $2 / 3$ & $2 / 3$ & $0 / 3$ & $0 / 3$ & $0 / 3$ & $0 / 3$ & $0 / 3$ & $0 / 3$ & $0 / 3$ & $0 / 3$ & $0 / 3$ & $0 / 3$ & $0 / 3$ & $0 / 3$ \\
\hline & $\begin{array}{l}\text { Nasal } \\
\text { swab }\end{array}$ & $0 / 3$ & $0 / 3$ & $0 / 3$ & $0 / 3$ & $0 / 3$ & $0 / 3$ & $0 / 3$ & $0 / 3$ & $0 / 3$ & $1 / 3$ & $1 / 3$ & $1 / 3$ & $0 / 3$ & $0 / 3$ & $0 / 3$ & $0 / 3$ & $0 / 3$ & $0 / 3$ \\
\hline & Tonsil & $0 / 3$ & $0 / 3$ & $0 / 3$ & $0 / 3$ & $0 / 3$ & $0 / 3$ & $1 / 3$ & $1 / 3$ & $1 / 3$ & $2 / 3$ & $2 / 3$ & $2 / 3$ & $1 / 3$ & $1 / 3$ & $1 / 3$ & $0 / 3$ & $0 / 3$ & $0 / 3$ \\
\hline & BAL & $0 / 3$ & $0 / 3$ & $0 / 3$ & $0 / 3$ & $0 / 3$ & $0 / 3$ & $0 / 3$ & $0 / 3$ & $0 / 3$ & $1 / 3$ & $1 / 3$ & $1 / 3$ & $0 / 3$ & $0 / 3$ & $0 / 3$ & $0 / 3$ & $0 / 3$ & $0 / 3$ \\
\hline & Urine & $0 / 3$ & $0 / 3$ & $0 / 3$ & $0 / 3$ & $0 / 3$ & $0 / 3$ & $0 / 3$ & $0 / 3$ & $0 / 3$ & $0 / 3$ & $0 / 3$ & $0 / 3$ & $0 / 3$ & $0 / 3$ & $0 / 3$ & $0 / 3$ & $0 / 3$ & $0 / 3$ \\
\hline & Feces & $0 / 3$ & $0 / 3$ & $0 / 3$ & $0 / 3$ & $0 / 3$ & $0 / 3$ & $0 / 3$ & $0 / 3$ & $0 / 3$ & $0 / 3$ & $0 / 3$ & $0 / 3$ & $0 / 3$ & $0 / 3$ & $0 / 3$ & $0 / 3$ & $0 / 3$ & $0 / 3$ \\
\hline \multirow{6}{*}{$\begin{array}{l}\text { ID/Prime } \\
\text { Pac (G3) }\end{array}$} & Serum & $0 / 3$ & $0 / 3$ & $0 / 3$ & $0 / 3$ & $0 / 3$ & $0 / 3$ & $0 / 3$ & $0 / 3$ & $0 / 3$ & $0 / 3$ & $0 / 3$ & $0 / 3$ & $0 / 3$ & $0 / 3$ & $0 / 3$ & $0 / 3$ & $0 / 3$ & $0 / 3$ \\
\hline & $\begin{array}{l}\text { Nasal } \\
\text { swab }\end{array}$ & $0 / 3$ & $0 / 3$ & $0 / 3$ & $0 / 3$ & $0 / 3$ & $0 / 3$ & $0 / 3$ & $0 / 3$ & $0 / 3$ & $0 / 3$ & $0 / 3$ & $0 / 3$ & $0 / 3$ & $0 / 3$ & $0 / 3$ & $0 / 3$ & $0 / 3$ & $0 / 3$ \\
\hline & Tonsil & $0 / 3$ & $0 / 3$ & $0 / 3$ & $0 / 3$ & $0 / 3$ & $0 / 3$ & $0 / 3$ & $0 / 3$ & $0 / 3$ & $0 / 3$ & $0 / 3$ & $0 / 3$ & $0 / 3$ & $0 / 3$ & $0 / 3$ & $0 / 3$ & $0 / 3$ & $0 / 3$ \\
\hline & BAL & $0 / 3$ & $0 / 3$ & $0 / 3$ & $0 / 3$ & $0 / 3$ & $0 / 3$ & $0 / 3$ & $0 / 3$ & $0 / 3$ & $0 / 3$ & $0 / 3$ & $0 / 3$ & $0 / 3$ & $0 / 3$ & $0 / 3$ & $0 / 3$ & $0 / 3$ & $0 / 3$ \\
\hline & Urine & $0 / 3$ & $0 / 3$ & $0 / 3$ & $0 / 3$ & $0 / 3$ & $0 / 3$ & $0 / 3$ & $0 / 3$ & $0 / 3$ & $0 / 3$ & $0 / 3$ & $0 / 3$ & $0 / 3$ & $0 / 3$ & $0 / 3$ & $0 / 3$ & $0 / 3$ & $0 / 3$ \\
\hline & Feces & $0 / 3$ & $0 / 3$ & $0 / 3$ & $0 / 3$ & $0 / 3$ & $0 / 3$ & $0 / 3$ & $0 / 3$ & $0 / 3$ & $0 / 3$ & $0 / 3$ & $0 / 3$ & $0 / 3$ & $0 / 3$ & $0 / 3$ & $0 / 3$ & $0 / 3$ & $0 / 3$ \\
\hline \multirow[t]{6}{*}{ NoVac (G4) } & Serum & $0 / 3$ & $0 / 3$ & $0 / 3$ & $0 / 3$ & $0 / 3$ & $0 / 3$ & $0 / 3$ & $0 / 3$ & $0 / 3$ & $0 / 3$ & $0 / 3$ & $0 / 3$ & $0 / 3$ & $0 / 3$ & $0 / 3$ & $0 / 3$ & $0 / 3$ & $0 / 3$ \\
\hline & $\begin{array}{l}\text { Nasal } \\
\text { swab }\end{array}$ & $0 / 3$ & $0 / 3$ & $0 / 3$ & $0 / 3$ & $0 / 3$ & $0 / 3$ & $0 / 3$ & $0 / 3$ & $0 / 3$ & $0 / 3$ & $0 / 3$ & $0 / 3$ & $0 / 3$ & $0 / 3$ & $0 / 3$ & $0 / 3$ & $0 / 3$ & $0 / 3$ \\
\hline & Tonsil & $0 / 3$ & $0 / 3$ & $0 / 3$ & $0 / 3$ & $0 / 3$ & $0 / 3$ & $0 / 3$ & $0 / 3$ & $0 / 3$ & $0 / 3$ & $0 / 3$ & $0 / 3$ & $0 / 3$ & $0 / 3$ & $0 / 3$ & $0 / 3$ & $0 / 3$ & $0 / 3$ \\
\hline & BAL & $0 / 3$ & $0 / 3$ & $0 / 3$ & $0 / 3$ & $0 / 3$ & $0 / 3$ & $0 / 3$ & $0 / 3$ & $0 / 3$ & $0 / 3$ & $0 / 3$ & $0 / 3$ & $0 / 3$ & $0 / 3$ & $0 / 3$ & $0 / 3$ & $0 / 3$ & $0 / 3$ \\
\hline & Urine & $0 / 3$ & $0 / 3$ & $0 / 3$ & $0 / 3$ & $0 / 3$ & $0 / 3$ & $0 / 3$ & $0 / 3$ & $0 / 3$ & $0 / 3$ & $0 / 3$ & $0 / 3$ & $0 / 3$ & $0 / 3$ & $0 / 3$ & $0 / 3$ & $0 / 3$ & $0 / 3$ \\
\hline & Feces & $0 / 3$ & $0 / 3$ & $0 / 3$ & $0 / 3$ & $0 / 3$ & $0 / 3$ & $0 / 3$ & $0 / 3$ & $0 / 3$ & $0 / 3$ & $0 / 3$ & $0 / 3$ & $0 / 3$ & $0 / 3$ & $0 / 3$ & $0 / 3$ & $0 / 3$ & $0 / 3$ \\
\hline
\end{tabular}

Detection of PRRSV in clinical samples by PCR. The results of PRRSV detection by PCR from different clinical samples collected at different necropsy days from the treatment groups are summarized in Table 3. Similar to the results of virus isolation in cell culture, PRRSV detection by PCR was varied depending on the clinical samples of each necropsy day and showed positive results by the results of virus isolation regardless of the days of necropsy and vaccine used as well as there was no PRRSV RNA in feces of all the treatment groups. None of the clinical samples of the ID/Prime Pac (G3) and NoVac (G4) groups were detected for PRRSV RNA at any necropsy days. PRRSV RNA was detected in sera of $3 / 3,3 / 3$, and $2 / 3$ pigs in the IM/Ingelvac MLV (G1) group at 7 to 21 DPV, respectively. Meanwhile, sera of pigs in the IM/Prime Pac (G2) group were positive for PRRSV with $1 / 3$ and $2 / 3$ pigs at 7 and 14 DPV, respectively. Like PRRSV isolation results, nasal swab samples from pigs in the IM/Ingelvac MLV (G1) group were positive for PRRSV at 21, 28, 35, and 42 DPV with $1 / 3,2 / 3$, $2 / 3$, and $1 / 3$ pigs, respectively. Nasal swabs of pigs in the IM/Prime Pac (G2) group were positive for PRRSV at 21 and 28 DPV with $1 / 3$ and $1 / 3$ pigs detected, respectively. PRRSV was detected in the tonsils from pigs in the IM/Ingelvac MLV (G1) group at 21, 28, 35, and 42 DPV with 3/3,3/3, 2/3, and 1/3 pigs detected, respectively. For the BAL, pigs in the IM/Ingelvac MLV (G1) group were positive for PRRSV at 21 and 28 DPV with $1 / 3$ and $2 / 3$ pigs detected, respectively. While 1 out of 3 pigs in the IM/Prime Pac (G2) group had PRRSV positive in the BAL at 28 DPV only (Table 3). PRRSV was detected in the urine sample from a pig in the IM/Ingelvac MLV (G1) group at 35 DPV but the urine samples from the IM/Prime Pac (G2) group showed no PRRSV positive.

Seroconversion of sentinel pigs. None of the sentinel pigs introduced to the ID/Prime Pac (G3) and NoVac (G4) groups were seropositive. Sentinel pigs introduced to the IM/Ingelvac MLV (G1) group at 7, 14, 21, and 28 DPV were seroconverted. In contrast, the sentinel pig introduced to the IM/Prime Pac (G2) group only at $21 \mathrm{DPV}$ was seropositive. 


\section{Experiment B}

Clinical signs. Pigs in the IM/High (T1) and ID/High (T2) groups displayed the clinical respiratory disease associated with PRRSV. Meanwhile, the IM/Low (T3) and ID/Low (T4) groups showed low or no clinical symptoms.

PRRSV specific antibody response. Antibody response following challenge was a dose-dependent manner (Fig. 1B). The IM/High (T1) and ID/High (T2) groups had observable antibody levels at 14 DPC. Significantly increased antibody tiers were observed in both groups from 14 to 28 DPC. In contrast, PRRSVspecific antibody titers of the IM/Low (T3) and ID/Low (T4) groups were first detected at 28 DPC. The antibody levels of IM/High (T1) and ID/High (T2) groups were significantly higher than that of the IM/Low (T3) and ID/Low (T4) groups from 14 to 28 DPC (Fig. 1B).

Viremia. Following the challenge, PRRSV RNA in challenged groups with high doses was rapidly increased and reached peaks at 7 DPC then continually decreased to basal levels from 14 to 28 DPC, respectively (Fig. 6B). In contrast, PRRSV RNA in challenged groups with low doses had continually increased and reached peaks at 14 DPC then rapidly decreased to basal levels at 21 and 28 DPC, respectively. The IM/High (T1) and ID/High (T2) groups had significantly higher $(P<0.05)$ PRRSV RNA (range from $209.51 \pm 37.7$ to $237.60 \pm 47.17$ copies/ml) than that of the IM/Low (T3) and ID/Low (T4) groups at 7 DPC (range from $115.12 \pm 20.22$ to $120.53 \pm 45.28$ copies/ml). Meanwhile, PRRSV RNA in the IM/Low (T3) and ID/Low (T4) groups were significantly $(P<$ 0.05) higher (range from $32.21 \pm 4.21$ to $194.43 \pm 40.74 \mathrm{copies} / \mathrm{ml}$ ) than that of the IM/High (T1) and ID/High (T2) groups (range from $19.09 \pm 3.03$ to 97.00 \pm 19.14 copies/ml) at 14 and $21 \mathrm{DPC}$, respectively (Fig. 6B).

Seroconversion of sentinel pigs. None of the sentinel pigs injected with Diluvac Forte via ID route was seropositive (Table 4). In contrast, sentinel pigs injected via IM route had seroconversion from 14 to 28 DPI, regardless of doses of PRRSV challenge. At 14 DPI, 4 and 2 of 6 sentinel pigs of the IM/High (T1) and IM/Low (T3) groups were seropositive, respectively. All 6 sentinel pigs both IM/High (T1) and IM/Low (T3) groups were seropositive at 21 and 28 DPI.

Table 4

Seroconversion of sentinel pigs in experiment B (Exp B) as measured by ELISA. Sera were considered positive for PRRSV antibody if the S/P ratio was greater than 0.4 . Values expressed as the number of positive sentinel pig(s)/total number of sentinel pigs $(n=6)$.

\begin{tabular}{|c|c|c|c|c|c|c|}
\hline \multirow[t]{3}{*}{ Experiment B } & \multirow[t]{3}{*}{ Apparatus for Diluvac Forte injection } & \multicolumn{5}{|c|}{ Seroconversion of sentinel pigs after injected with Diluvac Forte } \\
\hline & & \multicolumn{5}{|c|}{ Days post-injection (DPI) } \\
\hline & & 0 & 7 & 14 & 21 & 28 \\
\hline IM/High (T1) & Conventional needle $\left(\mathrm{G} 18,1^{\prime \prime}\right)$ & $0 / 6$ & $0 / 6$ & $4 / 6$ & $6 / 6$ & $6 / 6$ \\
\hline ID/High (T2) & Needle-free (IDAL 3G) & $0 / 6$ & $0 / 6$ & $0 / 6$ & $0 / 6$ & $0 / 6$ \\
\hline IM/Low (T3) & Conventional needle $\left(\mathrm{G} 18,1^{\prime \prime}\right)$ & $0 / 6$ & $0 / 6$ & $2 / 6$ & $6 / 6$ & $6 / 6$ \\
\hline ID/Low (T4) & Needle-free (IDAL 3G) & $0 / 6$ & $0 / 6$ & $0 / 6$ & $0 / 6$ & $0 / 6$ \\
\hline NoChal (T5) & - & $0 / 6$ & $0 / 6$ & $0 / 6$ & $0 / 6$ & $0 / 6$ \\
\hline
\end{tabular}

\section{Discussion}

The studies illustrated above were conducted to investigate the safety of vaccination with two PRRSV-2 MLV, either by IM or ID routes, in terms of the immune response, vaccine virus distribution in tissues and organs, and shedding patterns to sentinel pigs. Following vaccination, it was demonstrated that different PRRSV-2 MLV had different outcomes of the induction of an immune response and safety. It was notable that there were differences in early antibody detection between two PRRSV-2 MLV. Prime Pac PRRS (PP) vaccinated pigs, especially in ID vaccinated pigs, showed delayed antibody response compared to that of Ingelvac PRRS MLV vaccinated pigs and IM PP vaccinated. However, an ELISA response has long been recognized for its un-relationship with protection, unlike a response measured by viral neutralization assay. However, major findings in the present study are the induction of IFN-y-SC and IL-10. The IFN- $y$-SC in the ID PP vaccinated group had significantly higher frequencies than the other two IM vaccinated groups following vaccination. Increased IL-10 production was observed in all vaccinated groups following vaccination, and the ID vaccinated group had significantly lowest IL-10 levels compared to that of both IM vaccinated groups. For the evaluation of the safety following vaccination, our findings showed that PP vaccinated pigs had a shorter viremic phase, decreased the frequency of PRRSV distribution in organs, and shortened virus shedding compared to that of the Ingelvac PRRS MLV vaccinated pigs. Interestingly, an ID vaccination was best able to shorten the viremic phase, reduced the frequency of PRRSV distribution in organs, and shorten virus shedding compared to that of the intramuscular route.

For the humoral immune response, the results of the present study demonstrated that the induction of the humoral immune response of PRRSV MLV was different, regardless of vaccination routes. In agreement with our previous study, antibody response against PRRSV following vaccination with PP was delayed which reached above the cut-off levels at $21 \mathrm{DPV}^{17}$. The differences in the induction of PRRSV specific antibody response as measured by ELISA between IM and ID vaccinated pigs and are in accordance with findings from previous studies ${ }^{20,25}$. The mechanisms responsible for the induction of humoral immune response were not fully understood but the specific virus isolate used for the vaccines might play an important role.

Regarding cell-mediated immune response, delivery of antigen through the intradermal route could induce T cell polarization via the Th1 pathway, favoring the induction of IFN- $\gamma$ which was observed in the present study. The ID-vaccinated pigs showed a significantly higher IFN- $\gamma$-SC than that of the IM-vaccinated pigs. These findings are in agreement with previous reports in which the ID-vaccinated pigs induce relatively more IFN- $\gamma$-SC than that of the IM-vaccinated pigs $^{14,20,25}$. One factor probable committing to this finding is the presence of skin-resident immune cells capable of sufficiently capture antigens directly from 
the skin. The skin is rich in professional antigen-presenting cells (APC), including epidermal Langerhans cells (LC) and dermal dendritic cells, which are known to relocate to draining lymph nodes and trigger immune responses ${ }^{27}$.

Another feasibility of higher IFN-y-SC in the ID-vaccinated groups could be due to the lower IL-10 production. Our results showed that the IL-10 production was delayed, and the concentration was lower in ID-vaccinated pigs than in the IM-vaccinated pigs following vaccination. These findings are in agreement with previous reports in which both ID- and IM-vaccination induce IL-10 production, but the ID-vaccinated pigs induced comparably lower IL-10 levels than that of the IM-vaccinated pigs ${ }^{20,25}$. Nevertheless, the delivery of antigen through the intradermal route could target dendritic cells. IL-10 is a cytokine of the Th2 response. The delivery through this route could induce T cell polarization through the Th1 pathway, favoring other cytokines that act against Th2 ${ }^{28}$. The differences in IL-10 production among the PRRSV MLV vaccination group are not surprising. Previous reports demonstrated that PRRSV isolates vary in the degree of IL-10 production both in vivo and in vitro ${ }^{29,30}$. Additionally, the induction of IL-10 might depend on the virus isolate used in the experiment and the vaccine $\mathrm{v}^{31-33}$. However, the mechanism of IL-10 induction following vaccination by IM and ID routes are not understood.

The results of the present study demonstrated that some differences can be established in the safety of the 2 PRRSV MLV compared, although not all parameters were evaluated. Consequently, no differences in the induction of clinical signs were observed among vaccinated groups. Also, no clinical signs were recorded for any pig throughout the experiment. Although the affected lung lesion can be considered similar between vaccinated groups, the microscopic lung lesions increased over time until 28 DPV and resolved at 35 DPV, respectively (Fig. 5). This finding is simultaneous with previous reports in which indicate that lung lesions generated by PRRSV-2 MLV isolates tend to be more persistent and last longer than those produced by PRRSV-1 MLV isolates ${ }^{9,34}$. Therefore, it is assuring that the patterns of lung lesions observed in the present study are more relevant to the virus isolate used for the vaccine than the changes occurring through the attenuation process ${ }^{9}$. Nonetheless, since the pathophysiological characteristics of virus isolates used for the vaccine could not be determined in the present study, the impact of the attenuation process in the occurrence and progression of lung lesions cannot completely rule out.

Our results showed that the microscopic lung lesions of pigs vaccinated with Ingelvac PRRS MLV were significantly higher compared to PP vaccinated pigs on 21 DPV. These findings might be due to the difference in the intrinsic characteristics of the parent strains and, in particular, in their pneumotropism and pathological effects in the host might be related to these differences, that have been previously described ${ }^{23,35}$. Contrarily, the attenuation process might have altered the tropism of the vaccine viruses and restricted their ability to replicate in the lungs, and caused lung pathology. Remarkably, the frequency of virus isolation from the lung obtained in the present study was relatively lower than that of the other studies carried out with wide-type viruses ${ }^{34,36}$, indicating that the attenuation process of the vaccine virus might affect the cell tropism in the lung.

In addition to their ability to induce an immune response, the shedding patterns of PRRSV-2 MLV were evaluated using three different measurements, including the duration of viremia, the distribution of vaccine virus in organs, and the infection in sentinel pigs. Following vaccination, differences in the magnitude of viremia and the percentages of PRRSV-positive samples between PRRSV-2 MLV vaccinated groups were observed. Although the dynamics of viremia were similar among vaccinated groups, the magnitude of viremia of pigs vaccinated with Ingelvac PRRS MLV was statistically higher than those of the PP vaccinated pigs. In the same way, the percentage of PRRSV-positive tissue samples was higher in pigs vaccinated with Ingelvac PRRS MLV than in pigs vaccinated with the PP. These findings suggested that the viremic phase and the vaccine virus distribution in tissues were associated with the virus isolates used for the vaccines ${ }^{17}$. Notably, the ID-vaccinated pigs show no PRRSV-positive samples regardless of the detection analyses, although the magnitude of viremia of the ID-vaccinated pigs was similar to the IM-vaccinated pigs. The discrepancy between these finding could be due to the ability to replicate in the target cells of the vaccine virus beneath the skin tissues are limited. To postulate, additional studies are needed.

According to recent findings ${ }^{11}$, recombination events may occur between MLV and field strains of the virus. The reduction of MLV shedding post-vaccination may be able to reduce the potential of this recombination event occurring. Multiple other studies have also documented known vaccine strains recombining with field strains to form more pathogenic strains ${ }^{37}$. PRRSV is known to have a high degree of survivability in the environment ${ }^{38}$. Working on the assumption that survivability is similar to that of wild-type isolates, vaccine viruses shed into the environment may longer, be re-ingested by swine and play a part in new recombination isolates arising on the farm. However, we acknowledge that type of PRRSV MLV may have been attenuated through cell culture passage and hence have a decreased level of survivability in the environment. This will be the subject of a future study. Taken together, we postulate that a vaccine showing reduced shedding by vaccinated pigs of vaccine virus into the environment will reduce the risk of this happening significantly.

Supporting the case for intradermal needle-free vaccination, the Exp B illustrated the cessation of iatrogenic pathogen transfer between swine using a jet injector needle-free device (IDAL 3G), using PRRSV as a model in swine vaccinated with shared needles. Jet injection devices have been trialed in human medicine and utilize a high-pressure fluid jet, propelled by a spring or gases, to breach the skin, before introducing the vaccine antigen through a low-pressure jet $^{39}$. Theoretically, there is no possibility of pathogens being transferred from animal to animal using this pathway. However, it has been documented in the past that certain devices can transfer pathogens due to liquid splashing back into the device as the low-pressure stream ceases ${ }^{40,41}$. It is likely that differences exist across devices due to different design mechanics and that not all devices are hence equal. In conventional swine farming, needles are often shared between pigs that are being vaccinated. Needles shared in such a manner have been documented in human medicine to be capable of transmitting pathogens such as $\mathrm{HIV}^{42}$, and it is likely that for swine it is no different. Unfortunately, this is common practice on commercial swine operations due to the need for worker speed and efficiency. It is important to note that this study only assessed the potential for the vaccination 'jet' to transmit diseases and that great care was taken not to contaminate the device exterior between animals with fecal matter or other secretions. Given the highly transmissible nature of PRRSV in vivo ${ }^{16}$, it could be imagined that in a conventional situation, extraneous contamination of the devices may cause transmission of PRRSV via other routes, such as the oral-fecal route. 


\section{Conclusions}

Based on the present studies illustrated that different PRRSV-2 MLV had similar patterns of the induction of antibody response, but the differences were observed in the early phase following vaccination. The intradermal vaccination might represent an alternative to improve vaccine safety, as it induced lower IL-10 levels and more IFN-g-SC as well as the reduction of virus shedding within the herd and reduce the iatrogenic transfer of pathogens between animals with shared needles.

\section{Declarations}

\section{Acknowledgement.}

This study was supported by the Agricultural Research Development Agency (Public Organization) (grant number PRP6005020990), National Research Council of Thailand and Research and Researchers for Industries (grant number PHD59I0040), and the Second Century Fund (C2F), Chulalongkorn University. Furthermore, the partial funding was supported by Chulalongkorn University (CU) Special Task Force for Activation Research (STAR); swine virus evolution and vaccine research (SVEVR).

\section{Author Contributions.}

A.M., K.S.C., and D.N. designed experiment. A.M. and K.S.c. processed the samples and performed laboratory analyses. D.N. supervised the ex vivo experiment and performed pathological examinations and analyses. D.N. and A.T. supervised the statistical analysis. A.M., A.T. and D.N. drafted the manuscript and provided it through discussion and critical manuscript reading.

\section{Additional Information}

\section{Competing Interests.}

The authors declare no competing interests.

\section{References}

1. Mengeling, W. L., Lager, K. M. \& Vorwald, A. C. Clinical effects of porcine reproductive and respiratory syndrome virus on pigs during the early postnatal interval. Am. J. Vet. Res. 59, 52-55 (1998).

2. Done, S. H., Paton, D. J. \& White, M. E. Porcine reproductive and respiratory syndrome (PRRS): a review, with emphasis on pathological, virological and diagnostic aspects. Br. Vet. J. 152, 153-174. https://doi.org/10.1016/s0007-1935(96)80071-6 (1996).

3. Cavanagh, D. Nidovirales: a new order comprising Coronaviridae and Arteriviridae. Arch. Virol. 142, 629-633 (1997).

4. Kuhn, J. H. et al. Reorganization and expansion of the nidoviral family Arteriviridae. Arch. Virol. 161, 755-768. https://doi.org/10.1007/s00705-015-2672z (2016).

5. Stadejek, T., Stankevicius, A., Murtaugh, M. P. \& Oleksiewicz, M. B. Molecular evolution of PRRSV in Europe: current state of play. Vet. Microbiol. 165, 2128. https://doi.org/10.1016/j.vetmic.2013.02.029 (2013)

6. Collins, J. E. et al. Isolation of swine infertility and respiratory syndrome virus (isolate ATCC VR-2332) in North America and experimental reproduction of the disease in gnotobiotic pigs. J. Vet. Diagn. Invest. 4, 117-126. https://doi.org/10.1177/104063879200400201 (1992).

7. Shi, M. et al. Phylogeny-based evolutionary, demographical, and geographical dissection of North American type 2 porcine reproductive and respiratory syndrome viruses. J. Virol. 84, 8700-8711. https://doi.org/10.1128/JVI.02551-09 (2010)

8. Mengeling, W. L., Lager, K. M., Vorwald, A. C. \& Koehler, K. J. Strain specificity of the immune response of pigs following vaccination with various strains of porcine reproductive and respiratory syndrome virus. Vet. Microbiol. 93, 13-24. https://doi.org/10.1016/s0378-1135(02)00427-3 (2003).

9. Martinez-Lobo, F. J. et al. Safety of Porcine Reproductive and Respiratory Syndrome Modified Live Virus (MLV) vaccine strains in a young pig infection model. Vet. Res. 44, 115. https://doi.org/10.1186/1297-9716-44-115 (2013).

10. Mengeling, W. L., Vorwald, A. C., Lager, K. M. \& Brockmeier, S. L. Comparison among strains of porcine reproductive and respiratory syndrome virus for their ability to cause reproductive failure. Am. J. Vet. Res. 57, 834-839 (1996).

11. Wang, A. et al. Recombination between Vaccine and Field Strains of Porcine Reproductive and Respiratory Syndrome Virus. Emerg. Infect. Dis. 25, 23352337. https://doi.org/10.3201/eid2512.191111 (2019).

12. Weniger, B. G. \& Glenn, G. M. Cutaneous vaccination: antigen delivery into or onto the skin. Vaccine 31, 3389-3391. https://doi.org/10.1016/j.vaccine.2013.05.048 (2013).

13. Le Luduec, J. B. et al. Intradermal vaccination with un-adjuvanted sub-unit vaccines triggers skin innate immunity and confers protective respiratory immunity in domestic swine. Vaccine 34, 914-922. https://doi.org/10.1016/j.vaccine.2015.12.058 (2016).

14. Martelli, P. et al. Efficacy of a modified live porcine reproductive and respiratory syndrome virus (PRRSV) vaccine in pigs naturally exposed to a heterologous European (Italian cluster) field strain: Clinical protection and cell-mediated immunity. Vaccine 27, 3788-3799. https://doi.org/10.1016/j.vaccine.2009.03.028 (2009).

15. Cannon, J. E. et al. Pork quality audit: a review of the factors influencing pork quality 1. J. Muscle Foods 6, 369-402. https://doi.org/10.1111/j.17454573.1995.tb00581.x (1995). 
16. Pileri, E. \& Mateu, E. Review on the transmission porcine reproductive and respiratory syndrome virus between pigs and farms and impact on vaccination. Vet. Res. 47, 108. https://doi.org/10.1186/s13567-016-0391-4 (2016).

17. Madapong, A. et al. Humoral immune responses and viral shedding following vaccination with modified live porcine reproductive and respiratory syndrome virus vaccines. Arch. Virol. 162, 139-146. https://doi.org/10.1007/s00705-016-3084-4 (2017).

18. Nilubol, D., Tripipat, T., Hoonsuwan, T. \& Kortheerakul, K. Porcine reproductive and respiratory syndrome virus, Thailand, 2010-2011. Emerg. Infect. Dis. 18, 2039-2043. https://doi.org/10.3201/eid1812.111105 (2012).

19. Madapong, A., Saeng-Chuto, K., Boonsoongnern, A., Tantituvanont, A. \& Nilubol, D. Cell-mediated immune response and protective efficacy of porcine reproductive and respiratory syndrome virus modified-live vaccines against co-challenge with PRRSV-1 and PRRSV-2. Sci. Rep. 10, 1649. https://doi.org/10.1038/s41598-020-58626-y (2020).

20. Madapong, A. et al. Immune response and protective efficacy of intramuscular and intradermal vaccination with porcine reproductive and respiratory syndrome virus 1 (PRRSV-1) modified live vaccine against highly pathogenic PRRSV-2 (HP-PRRSV-2) challenge, either alone or in combination with of PRRSV-1. Vet. Microbiol. 244, 108655. https://doi.org/10.1016/j.vetmic.2020.108655 (2020).

21. Nilubol, D., Tripipat, T., Hoonsuwan, T., Tipsombatboon, P. \& Piriyapongsa, J. Genetic diversity of the ORF5 gene of porcine reproductive and respiratory syndrome virus (PRRSV) genotypes I and II in Thailand. Arch. Virol. 158, 943-953. https://doi.org/10.1007/s00705-012-1573-7 (2013).

22. Chaikhumwang, P. et al. Dynamics and evolution of highly pathogenic porcine reproductive and respiratory syndrome virus following its introduction into a herd concurrently infected with both types 1 and 2. Infect. Genet. Evol. 30, 164-174. https://doi.org/10.1016/j.meegid.2014.12.025 (2015).

23. Halbur, P. G. et al. Comparison of the pathogenicity of two US porcine reproductive and respiratory syndrome virus isolates with that of the Lelystad virus. Vet. Pathol. 32, 648-660. https://doi.org/10.1177/030098589503200606 (1995).

24. Halbur, P. et al. Efficacy of antimicrobial treatments and vaccination regimens for control of porcine reproductive and respiratory syndrome virus and Streptococcus suis coinfection of nursery pigs. J. Clin. Microbiol. 38, 1156-1160 (2000).

25. Ferrari, L. et al. Lymphocyte activation as cytokine gene expression and secretion is related to the porcine reproductive and respiratory syndrome virus (PRRSV) isolate after in vitro homologous and heterologous recall of peripheral blood mononuclear cells (PBMC) from pigs vaccinated and exposed to natural infection. Vet. Immunol. Immunopathol. 151, 193-206. https://doi.org/10.1016/j.vetimm.2012.11.006 (2013).

26. Nilubol, D., Platt, K. B., Halbur, P. G., Torremorell, M. \& Harris, D. L. The effect of a killed porcine reproductive and respiratory syndrome virus (PRRSV) vaccine treatment on virus shedding in previously PRRSV infected pigs. Vet. Microbiol. 102, 11-18. https://doi.org/10.1016/j.vetmic.2004.05.006 (2004).

27. Combadiere, B. \& Liard, C. Transcutaneous and intradermal vaccination. Hum. Vaccin. 7, 811-827. https://doi.org/10.4161/hv.7.8.16274 (2011).

28. Tesfaye, D. Y., Gudjonsson, A., Bogen, B. \& Fossum, E. Targeting Conventional Dendritic Cells to Fine-Tune Antibody Responses. Front. Immunol. 10, 1529. https://doi.org/10.3389/fimmu.2019.01529 (2019).

29. Diaz, I., Darwich, L., Pappaterra, G., Pujols, J. \& Mateu, E. Immune responses of pigs after experimental infection with a European strain of Porcine reproductive and respiratory syndrome virus. J. Gen. Virol. 86, 1943-1951. https://doi.org/10.1099/vir.0.80959-0 (2005).

30. Chung, H. K. \& Chae, C. Expression of interleukin-10 and interleukin-12 in piglets experimentally infected with porcine reproductive and respiratory syndrome virus (PRRSV). J. Comp. Pathol. 129, 205-212. https://doi.org/10.1016/s0021-9975(03)00036-7 (2003).

31. Diaz, I., Darwich, L., Pappaterra, G., Pujols, J. \& Mateu, E. Different European-type vaccines against porcine reproductive and respiratory syndrome virus have different immunological properties and confer different protection to pigs. Virology 351, 249-259. https://doi.org/10.1016/j.virol.2006.03.046 (2006).

32. Silva-Campa, E. et al. European genotype of porcine reproductive and respiratory syndrome (PRRSV) infects monocyte-derived dendritic cells but does not induce Treg cells. Virology 396, 264-271. https://doi.org/10.1016/j.virol.2009.10.024 (2010).

33. Silva-Campa, E. et al. Induction of T helper 3 regulatory cells by dendritic cells infected with porcine reproductive and respiratory syndrome virus. Virology 387, 373-379. https://doi.org/10.1016/j.virol.2009.02.033 (2009).

34. Martinez-Lobo, F. J. et al. Comparative pathogenicity of type 1 and type 2 isolates of porcine reproductive and respiratory syndrome virus (PRRSV) in a young pig infection model. Vet. Microbiol. 154, 58-68. https://doi.org/10.1016/j.vetmic.2011.06.025 (2011).

35. Halbur, P. G. et al. Comparison of the antigen distribution of two US porcine reproductive and respiratory syndrome virus isolates with that of the Lelystad virus. Vet. Pathol. 33, 159-170. https://doi.org/10.1177/030098589603300205 (1996).

36. Rossow, K. D. et al. Pathogenesis of porcine reproductive and respiratory syndrome virus infection in gnotobiotic pigs. Vet. Pathol. 32, 361-373. https://doi.org/10.1177/030098589503200404 (1995).

37. Li, B. et al. Recombination in vaccine and circulating strains of porcine reproductive and respiratory syndrome viruses. Emerg. Infect. Dis. 15, $2032-2035$. https://doi.org/10.3201/eid1512.090390 (2009).

38. Dee, S. A., Martinez, B. C. \& Clanton, C. Survival and infectivity of porcine reproductive and respiratory syndrome virus in swine lagoon effluent. Vet. Rec. 156, 56-57. https://doi.org/10.1136/vr.156.2.56 (2005).

39. Verhagen, A., Ebels, J. T., Dogterom, A. A. \& Jonkman, J. H. Pharmacokinetics and pharmacodynamics of a single dose of recombinant human growth hormone after subcutaneous administration by jet-injection: comparison with conventional needle-injection. Eur. J. Clin. Pharmacol. 49, 69-72. https://doi.org/10.1007/BF00192361 (1995).

40. Baxter, J. \& Mitragotri, S. Needle-free liquid jet injections: mechanisms and applications. Expert Rev. Med. Devices 3, 565-574. https://doi.org/10.1586/17434440.3.5.565 (2006).

41. Brink, P. R., van Loon, A. M., Trommelen, J. C., Gribnau, F. W. \& Smale-Novakova, I. R. Virus transmission by subcutaneous jet injection. J. Med. Microbiol. 20, 393-397. https://doi.org/10.1099/00222615-20-3-393 (1985).

Page $11 / 16$ 
42. Ball, L. J. et al. Sharing of Injection Drug Preparation Equipment Is Associated With HIV Infection: A Cross-sectional Study. J. Acquir. Immune. Defic. Syndr. 81, e99-e103. https://doi.org/10.1097/QAl.0000000000002062 (2019).

\section{Figures}

(A) PRRSV-specific antibody response as measured by IDEXX ELISA

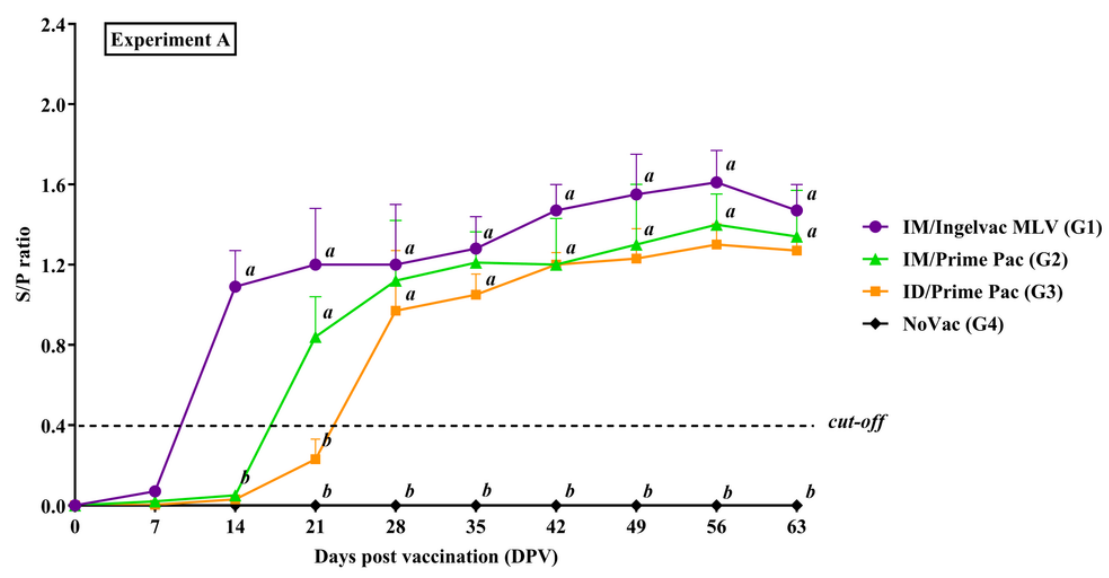

(B) PRRSV-specific antibody response as measured by IDEXX ELISA

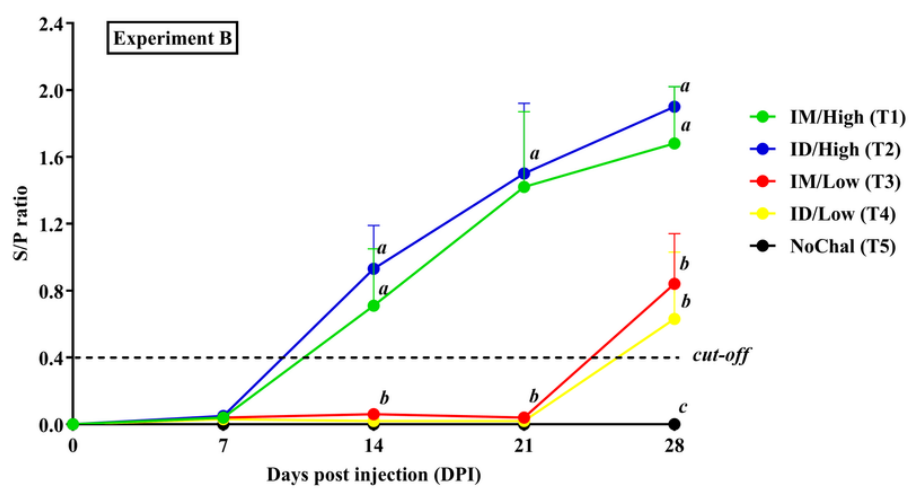

Figure 1

PRRSV-specific antibodies of pigs in the $\operatorname{Exp} A(A)$ or Exp B (B) measured by ELISA. Values expressed as mean \pm SEM. Sample-to-positive (S/P) ratios equal to or greater than 0.4 (dash-line). The results were compared using two-way ANOVA for multiple comparisons. Different lowercase letters (a-b) indicate significant differences between treatment groups $(P<0.05)$ for each day. 


\section{Porcine IL-10 production}

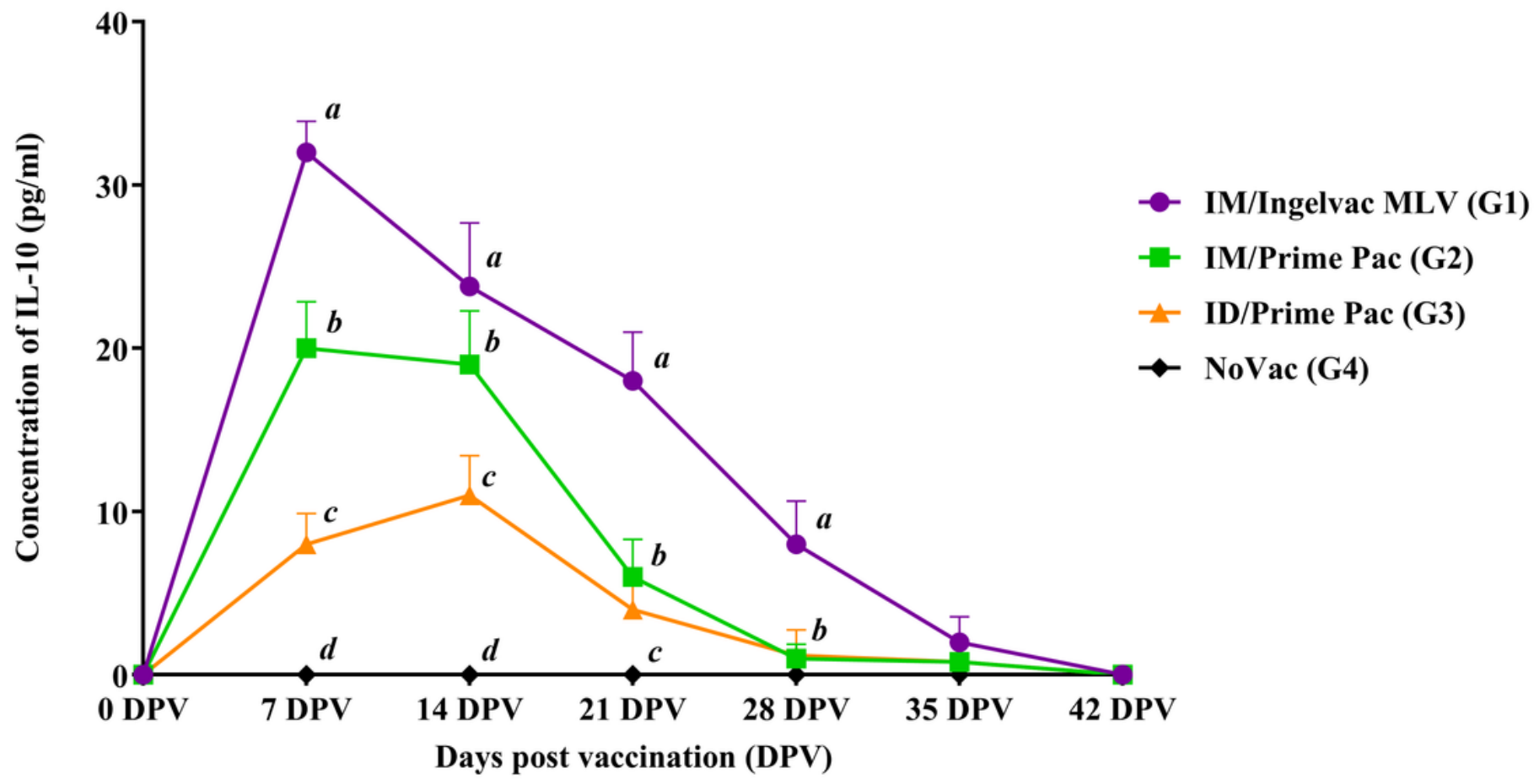

Figure 2

Quantification of porcine IL-10 in stimulated PBMC with homologous viruses (vaccine viruses) of pigs in the Exp A. Values expressed as mean \pm SEM. The results were compared using two-way ANOVA for multiple comparisons. Different lowercase letters (a-b) indicate significant differences between treatment groups $(P<0.05)$ for each day.

PRRSV-specific IFN- $\gamma$-SC against homologous viruses (vaccine viruses)

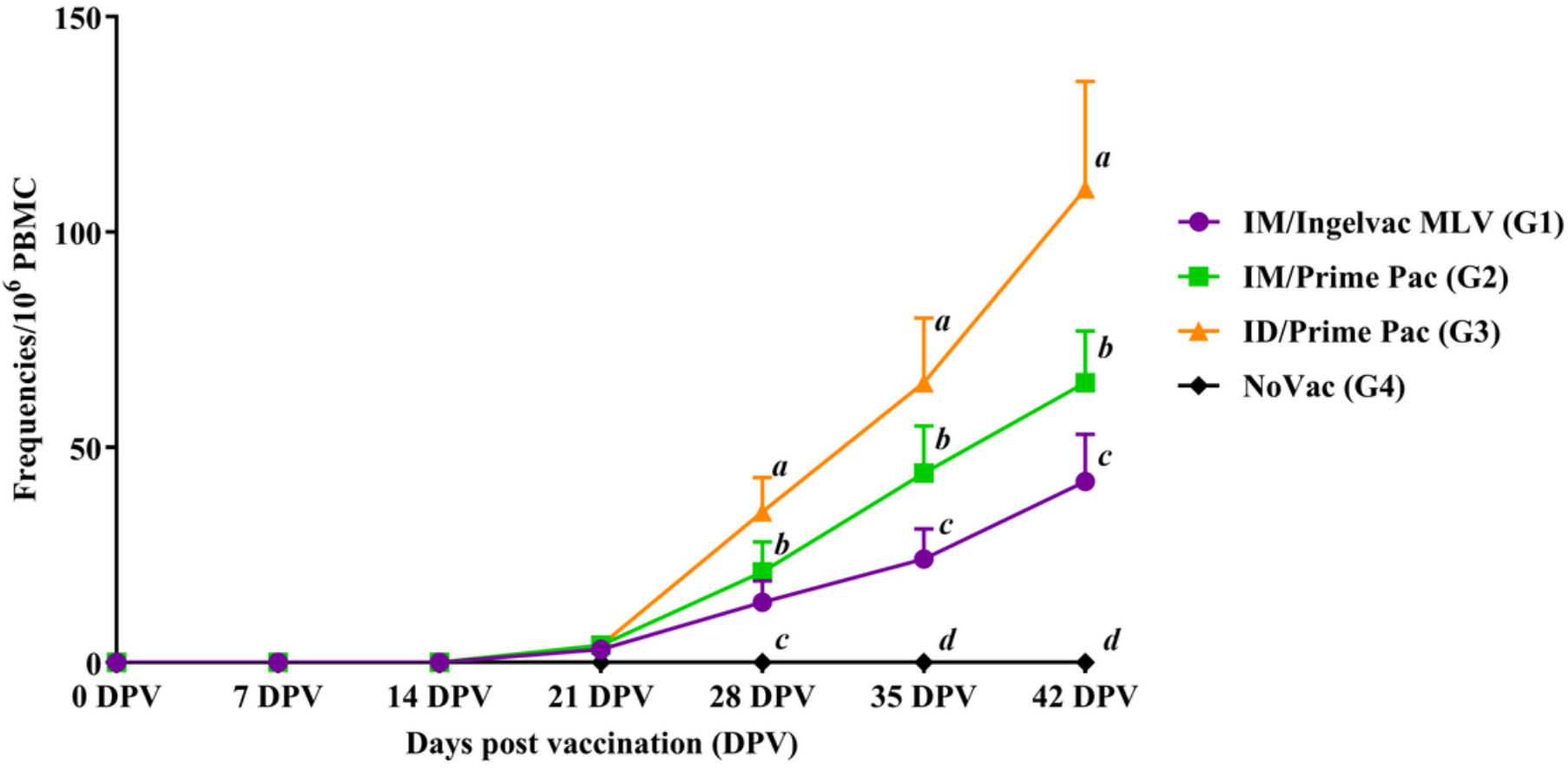



compared using two-way ANOVA for multiple comparisons. Different lowercase letters $(a-b)$ indicate significant differences between treatment groups $(P<$

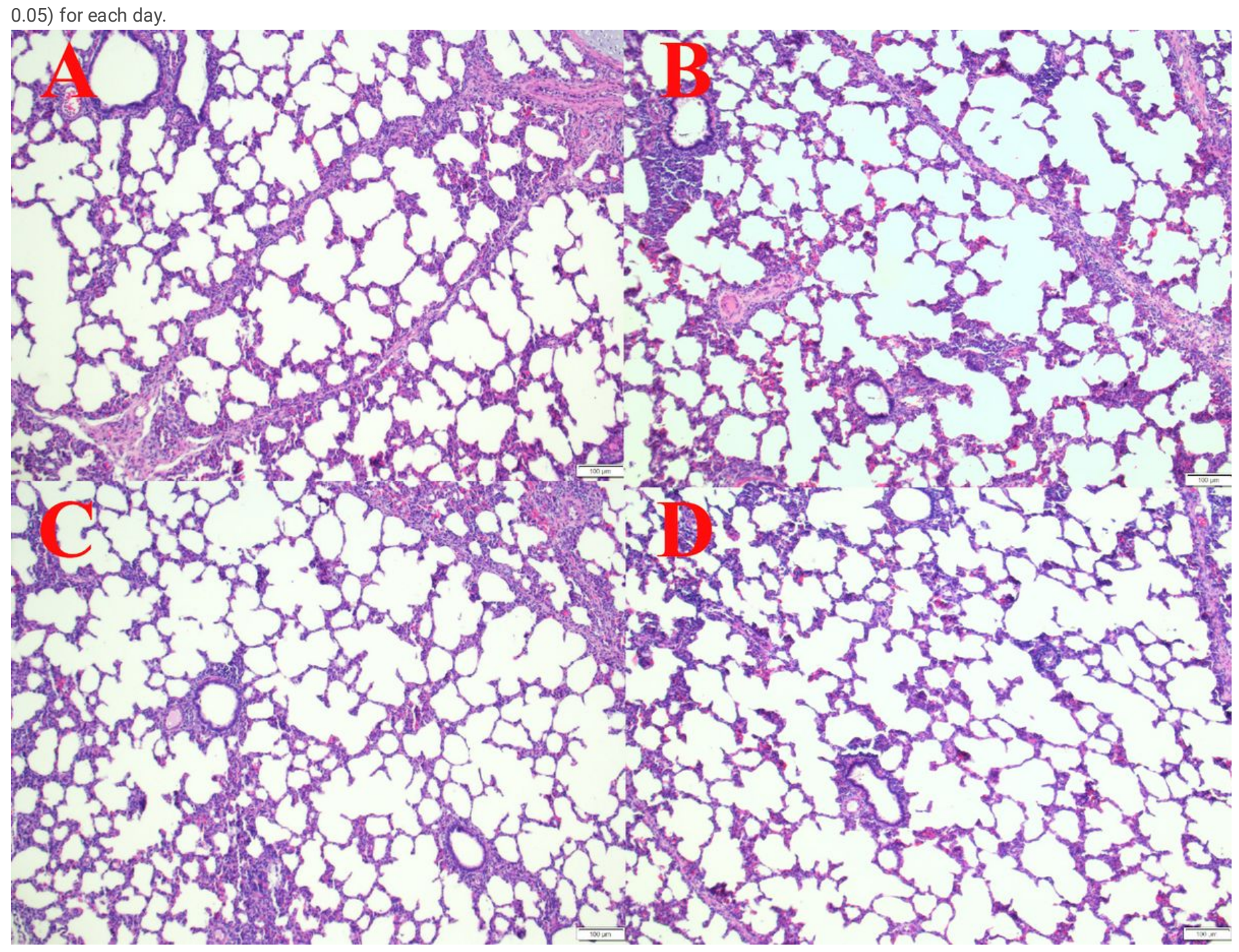

Figure 4

Microscopic lung lesion of pigs in the Exp A; (A) IM/Ingelvac MLV (G1), (B) IM/Prime Pac (G2), (C) ID/Prime Pac (G3), and (D) NoVac (G4) groups, respectively. H\&E staining. Bar $=100 \mu \mathrm{m}$. 


\section{Microscopic lung lesion scores}

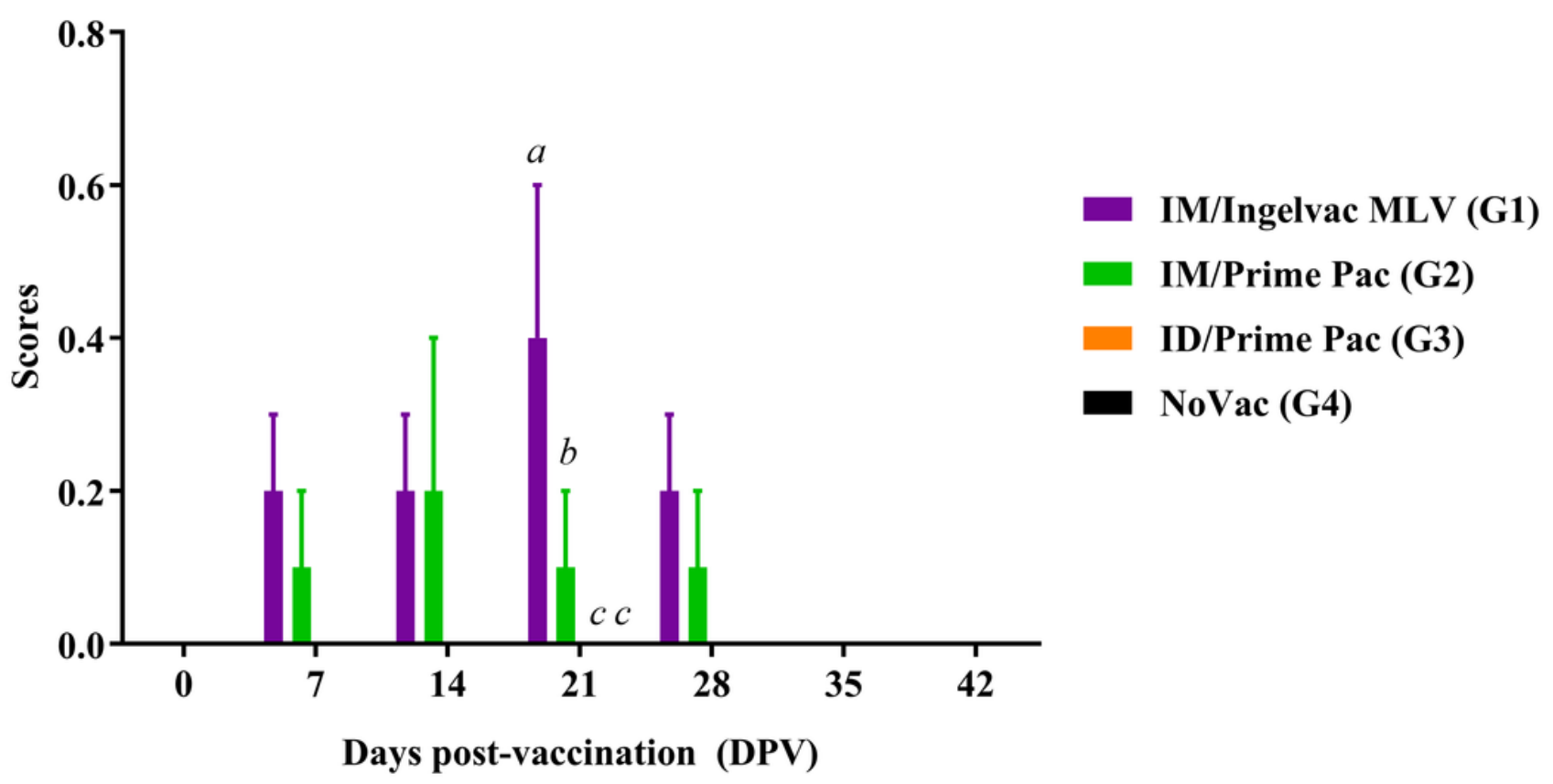

Figure 5

The mean values of the microscopic lung lesion scores of pigs in the Exp A following vaccination. Values expressed as mean \pm SEM. The results were compared using two-way ANOVA for multiple comparisons. Different lowercase letters $(a-c)$ indicate significant differences between treatment groups $(P<$ 0.05) for each day. 
(A)
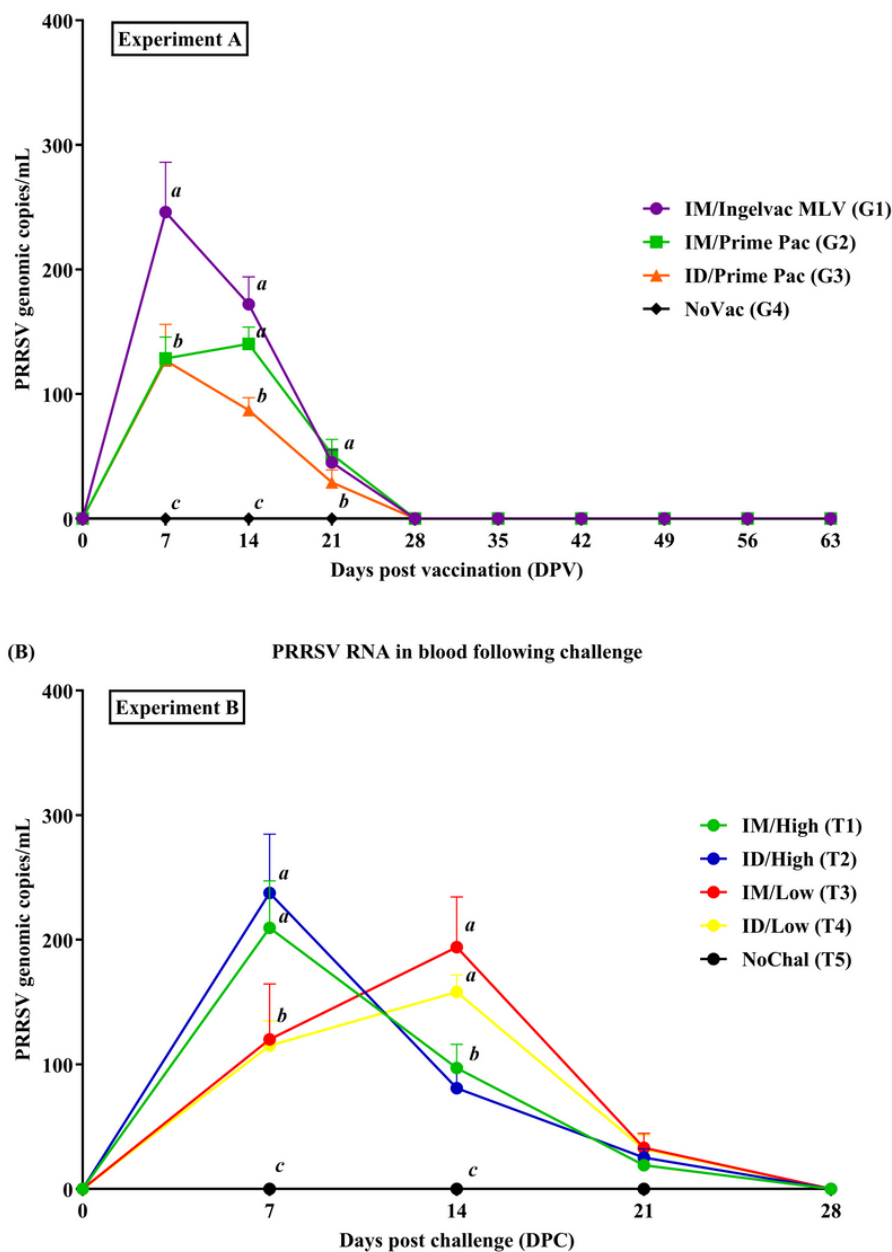

Figure 6

Mean genomic copy number of PRRSV RNA in the blood of pigs following vaccination in the Exp A (A) or following challenge in the Exp B (B). Values expressed as mean \pm SEM. The results were compared using two-way ANOVA for multiple comparisons. Different lowercase letters (a-c) indicate significant differences between treatment groups $(P<0.05)$ for each day. 